\title{
Rekole 14_006 ou le fiasco de la gestion hospitalière
}

\section{Olivier Despond}

Dr méd., membre de la FMH

\section{"Aucune avancée n'est plus difficile que le retour à la raison."}

Berthold Brecht

Depuis bientôt 20 ans, je supervise la saisie des données statistiques des prestations d'anesthésie de notre service. Ce travail de récolte, basé sur des données généralement bien définies et relativement simples, a permis pendant de nombreuses années à notre service et à tout son personnel de suivre l'évolution de nos prestations, de remplir les livrets de formation de nos médecins assistants, de réaliser toute la facturation, de permettre un contrôle de qualité interne objectif et efficace ainsi que de participer au projet ADS (Anes-

\section{L'introduction de cette dernière variable pose de grands problèmes à tout notre personnel.}

thésie Données Suisse) regroupant un quart des services d'anesthésie de Suisse, permettant entre autres un benchmarking plus qu'intéressant grâce aux analyses fines et judicieuses des professionnels de l'Institut Universitaire de Médecine Sociale et Préventive de Lausanne (IUMSP). Tout ceci pour un coût relativement modique.

A part le groupe ADS, peu de monde hélas s'est intéressé à ces données, malgré un besoin croissant et évident de données fiables, à quelque niveau que ce soit.

Les données que nous saisissons sont très fiables, raison pour laquelle elles servent entre autres à la validation Rekole de notre hôpital sur les données opératoires, permettant une facturation optimale. En effet nous saisissons depuis très longtemps les items temporels chronologiques, à savoir:

$\mathrm{X}$ le début de l'anesthésie

$\Delta$ la fin de l'induction de l'anesthésie, patient à disposition de l'opérateur

$\Theta$ le début de l'intervention

$\Theta$ la fin de l'intervention

$\Delta$ la remise du patient à l'anesthésiste

$\mathrm{X}$ la fin de l'anesthésie

$\mathrm{V}$ le départ de la salle de réveil

\section{Une exactitude enviable}

Dans beaucoup d'hôpitaux, il appartient aux anesthésistes de saisir ces items, car eux seuls suivent d'un bout à l'autre le cheminement du patient, et c'est peut-être grâce à l'esprit pointilleux de l'anesthésiste (qui entre autres gère les médicaments au microgramme près) que ces données sont d'une exactitude enviable, pour peu qu'une personne bienveillante ait un regard global sur ces données. De plus si ces données sont fiables, c'est dû au fait que leur définition est simple et claire, le $\mathrm{X}$ début de l'anesthésie est effectivement et empiriquement un moment évident pour tout le personnel d'anesthésie. Etant membre du Comité de la Société Suisse d'Anesthésiologie et Réanimation, responsable des tarifs, c'est peu dire que l'exactitude de données temporelles est importante pour la tarification, en particulier pour le TARMED, qui prévoit entre autres des durées opératoires, de préparation et de finition. J'ai souvent pu faire des comparaisons entre mes chiffres et ceux proposés dans le TARMED pour évaluer notre efficacité ou au contraire notre manque d'efficacité.

\section{Le fiasco futur prévisible}

Evolution oblige, une nouvelle variable temporelle a été introduite début 2016. En effet le début de l'anesthésie $\mathrm{X}$ est devenu: $\mathrm{X}$ la prise en charge du patient par l'anesthésie, et la nouvelle variable $\Omega$ : «début de l'induction de l'anesthésie» est apparue, définie comme «l'heure à laquelle les premières mesures anesthésiologiques sont initiées», ou alors "le moment de l'administration des premiers médicaments». Nous en sommes donc actuellement à 8 variables temporelles à saisir et j'ai pu, au fil des mois, constater que l'introduction de cette dernière variable $\Omega$ pose de grands problèmes à tout notre personnel pourtant discipliné, car cet $\Omega$ ne reflète pas un instant clair et simple dans le cheminement du patient. Par conséquent, on en vient à introduire une donnée le plus souvent inexacte (5 minutes systématiquement?), qui ne sert en plus à rien du point de vue tarifaire, le patient étant déjà sous la responsabilité de l'anesthésiste. 
Soit! J'en viens au fiasco futur prévisible mentionné plus haut. Sur proposition de SwissDRG, et de trois hôpitaux de l'est de la Suisse, la commission d'expert Rekole des Hôpitaux de Suisse H+ a décidé (proposition 14_006) de l'obligation de saisir trois items temporels

\section{Il est déjà évident que ces items seront soit erronés, soit inutiles dans la majorité des cas.}

supplémentaires dès 2018, à savoir le «début de la préparation» $\mathrm{P}$ du patient (rasage, positionnement), «l'entrée en salle d'opération» et «le début des préparations» D (mesures chirurgicales préparatoires sur le patient). Et ceci sans compter d'autres temps qui seraient à saisir à l'avenir, soit... le moment de la prémédication, le moment de l'appel pour amener le patient au bloc opératoire, la fin du réveil du patient, la fin de la sortie de salle d'opération, voire la fin de la transmission des documents.

Bref, on s'achemine vers la saisie de 17 ou 18 items temporels, pour satisfaire les ambitions d'experts en comptabilité et en gestion.

\section{Dilapidation pour de l'inutile}

Il est d'ores et déjà évident, vu la difficulté de définir ces nouveaux items, et surtout la variabilité importante dans la prise en charge des patients en fonction des hôpitaux (qui ne sont pas des usines avec des algorithmes de soins, malgré ce que continuent de croire certains), que ces items seront soit erronés, soit inutiles dans la majorité des cas. Qui, avec un patient éventuellement instable, va s'occuper de saisir des données qui sembleront futiles à la majorité des protagonistes de la santé? Va-t-on engager une personne rien que pour cette fonction? Va-t-on comparer des hôpitaux qui font une désinfection en présalle avec ceux qui ne font une désinfection qu'en salle? Quid des interventions en secteur blanc (dentiste, endoscopie, etc...)?
Le simple fait de lire au complet la proposition 14_006 provoque le vertige et la démotivation. Je cite juste un exemple: «lors de la détermination du facteur de simultanéité (FS), le fait qu'un médecin arrive plus tard ou s'en va plus tôt est pris en compte. Le temps effectif de présence du médecin est saisi. Des durées prédéfinies peuvent être appliquées dans ce contexte.»

Ainsi donc une Commission d'expert REKOLE de H+ a décidé à l'unanimité de forcer les médecins ou du personnel à saisir des données qui seront forcément inexactes et donc ininterprétables sous prétexte que

\section{Le simple fait de lire la proposition 14_006 provoque le vertige et la démotivation.}

le Casemix Office de SwissDRG pense que la procédure de calcul des coûts en salle d'opération pourrait (pourrait!) être améliorée. Personne ne pose la question du coût informatique et en personnel pour la saisie de ces variables.

La SSAR a validé le Projet national de données statistiques et contrôle de qualité A-QUA, pour l'anesthésie, projet déjà difficile à mettre sur pied, car il est ambitieux. Obtenir des données fiables est déjà un challenge en soi, mais le fait qu'il soit alourdi par l'obligation de saisir des données supplémentaires futiles et déplacées est vraiment démotivant.

Il est temps que les responsables de la santé cessent d'avaliser n'importe quel projet, sous prétexte que plus d'informations égale plus de qualité ou d'efficience. On lutte contre l'explosion des coûts, mais on dilapide massivement pour de l'inutile.

Nous avons besoin de données fiables, simples, explicites.

\section{Référence}

http://www.hplus.ch/fileadmin/user_upload/H__Verband/ Fachkommission/REK/14_006_pub_f.pdf 